\title{
New approaches to safety screening
}

\section{By D. M. Conning, The British Industrial Biological Research Association, Woodmansterne Road, Carshalton, Surrey $S_{5}{ }_{4} D S$}

There can be no doubt that health and safety are now topics of concern that involve most human activities, and the relative safety of foodstuffs is among the most important. Hitherto, in this country, we have been particularly concerned with the food chemicals we use to exact the most economic benefit from available supplies, but it is increasingly being realized that many natural components, contaminants or breakdown products may make measurable contributions to potential hazard (Salunkhe $\& \mathrm{Wu}$, 1977).

The practice of toxicology has undergone remarkable technical advance throughout the past few decades. Not only has the range of biological problems identified as having a toxicological component increased, but the available analytical techniques have allowed an extraordinary range of measurements to be made, and to smaller and smaller limits of detection. Thus the technical complexity of toxicological investigation has reached an extraordinary pitch, with the concomitant increase in relative costs (Waitt, 1975).

The control of health and safety issues has fallen upon government departments, either specially established for the purpose or which have developed from existing facilities. As our ability to exchange information has increased, so has the development of international coordinating mechanisms proceeded, and the system of legislative control is now simply enormous. This has resulted in very substantially increased demands being made on industrialists who wish to market products in any of the areas covered.

At an early stage in the development of regulatory control it was realized that the essence of the problem was the extrapolation of experimental animal results to the human situation, given the known variation in species response to different chemicals, the known impact of dosage on toxic response and the indeterminate nature of the relationship between acute and chronic administration. Three principles arose from this; first, that a lifetime of administration in the experimental animal could be assumed to equate with a lifetime of exposure in man; secondly, that the assertion of safety should be based on an absence of detectable effect rather than a containment of the effect; and thirdly, that further safety factors should be applied to ensure no adverse consequences of possibly increased human sensitivity. Thus was born the concept of 'no-effect levels' and, by application of safety factors, the concept of 'acceptable daily intake'--and also, incidentally, the famous Delaney amendment which, in effect, ensured that the ADI was zero for experimentally proven oral carcinogens.

The consequence of this approach to legislation has been a system which, though complex, is easily administered by personnel who are not practising 
toxicologists. It achieves a fair degree of safety within the confines of known parameters but, conversely, ignores the possibility of detecting hazards not recognized as such. It is very expensive in terms of money, animals and human resources, and adds nothing to our understanding of basic biological processes or toxicological mechanisms. In addition, there have been claims that the imposition of such regulatory burdens has imposed a serious handicap on the development of the chemical industry, especially in its more specialized forms such as pharmaceuticals or agrochemicals.

Given these adverse consequences, the time is appropriate to reconsider our basic approach and perhaps to consider a system more in tune with the needs of society and industry. Such a system would not seek to ensure an absence of hazard based on its identification at any dosage, but would seek to ensure an absence of hazard at the intended level of use. It would also seek to detect such hazard by an examination of the specific biological processes affected.

If we confine our attention to food chemicals and do not consider handling hazards applicable to manufacturing processes, i.e. consider only the hazards to the consumer, a number of questions present themselves which may be arranged as an algorithm (Fig. I). (I) Is anything known about this chemical or chemicals of this type that allows us to predict toxicity? There is an increasing amount of information which could be worked into programmes for narrowing down areas of ignorance about hazardous chemicals. The whole topic of structure-function analyses is ripe for a concerted attack based on accumulated experience (Cramer et al. 1978). (2) Is there detectable absorption at intended levels of exposure and, if so, is there distribution or accumulation in tissues known to be vulnerable? Tissues such as bone marrow, central nervous system and germinal epithelium are assumed to be of greater importance as target tissues than, for example, liver and kidney which have substantial reserves and powers of regeneration. If such localization can be detected, or if the half-life of the material is prolonged, subacute studies should be undertaken. Similarly, if a chemical is absorbed, placental localization or transfer should be measured, and if it occurs some form of teratological screen undertaken. If absorption does not occur, there remains the possibility of local gut damage. (3) Subacute studies cannot give an indication of potential carcinogenesis that might be present on long-term administration. There is now a well established, though not effectively evaluated, screening procedure for determining the ability of a chemical to interact with DNA to provoke mutation, utilizing bacteria (McCann et al. 1975). Such tests do not give any information on the potency of the reaction or its relevance to mammalian systems. They are capable of detecting those circumstances where a compound which covalently binds with DNA, damages that DNA to an extent which could cause mutation if adequate repair did not occur. They should therefore be preceded by some assessment of the ability of the suspect compound to bind covalently with mammalian DNA, that is to form macromolecular adducts. If such binding occurs, then it would be appropriate to determine if adverse consequences could ensue by using the Ames test or similar procedure. (4) Finally, there remains the possibility 

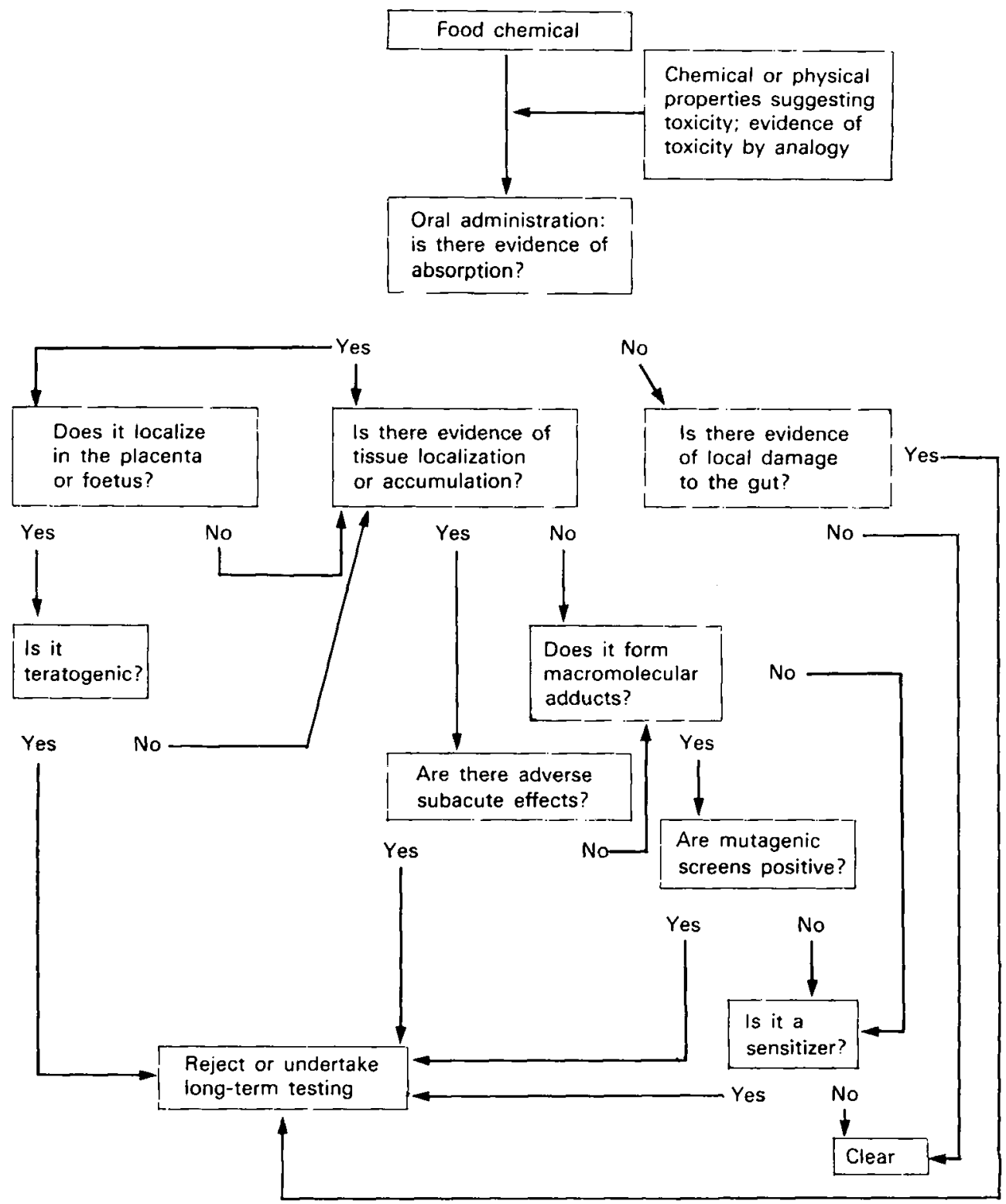

Fig. I. Schematic representation of a system for the investigation of a food chemical.

that a compound may induce some kind of immunological response, or might be instrumental in inhibiting or distorting immunological mechanisms, and this should be looked for specifically.

The scheme as presented here includes no indication of the methodology to be used because this is subject to the results of further research. It is clear that there is not enough information available at this time to establish this approach as a regulatory procedure, but the principle on which it is based-namely that the 
toxicity of a food chemical is related to the rate of absorption and to the tissue concentrations, and that these depend in part on the amounts in food-could be accepted on the basis of current knowledge.

Further research is needed to establish the procedures based on an understanding of some of the mechanisms involved. It is neither necessary nor possible to base any regulatory procedure on complete elucidation of toxicological mechanisms, but it should be possible to identify key events that are recognized as crucial to the development of the disease process under consideration.

Some areas of research which then present themselves include; ( $\mathrm{I}$ ) structure-function analysis: this might be nothing more than a process of accumulating information and systems of analysis, but it seems possible that enough is now known about many toxicological processes to be able to make positive associations between molecular configuration and adverse pharmacological or biochemical consequences. (2) The feasibility of low dosage pharmaco-kinetics and its predictability from high dose studies. This includes the relationship between dosage and tissue distribution and metabolism. There is the related topic of repeated dosage on a subacute basis (say $\mathrm{I}_{4}-28 \mathrm{~d}$ ), the possibility of accumulation and related toxicity. Is there a relationship between dose and duration in promoting tissue pathology? (3) The relationship between placental localization or transfer, foetal pharmaco-kinetics and teratology. (4) Is it a feasible proposition that electrophilic compounds that do not covalently bind with macromolecules, cannot cause damage to DNA? Is there a relationship between this and the inhibition of DNA repair? Is it possible that reactivity with DNA is a very simple and common phenomenon, and that it is the consequent control of this which is the important factor in mutagenesis or carcinogenesis? (5) What is the optimum method for detecting that an immune reaction is in progress? Should we be looking at populations of specialized lymphocytes or is it simply enough to weigh the thymus during a subacute study? Is there evidence that some chemicals can impede the immune process? Is this important?

The regulatory process embodied in this scheme would require several pieces of information: ( $\mathrm{r}$ ) at what concentration is the chemical to be used and what is the maximum ingestion to be expected by any section of the community? (2) Is there evidence of critical tissue damage at this level of exposure? This includes foetal tissues. (3) Is it a possible mutagen? (4) Is it immunologically active?

A positive answer to any of these questions could trigger longer term studies utilizing 'no effect levels' of a more specific type. Negative answers would result in clearance, to be reviewed at regular intervals. Whether present systems could be adapted to this scheme remains to be seen, but it seems possible, especially in the UK where there is a tradition of assessment by scientists rather than the widespread use of 'checklists'.

It seems likely that the proposed approach would result in better qualitative information on which to base an assessment of human hazard. There would be a series of positive or negative findings with no room for judgements in terms of hazardous dosage. This is essentially because the initial testing is done at levels 
which relate to levels of use, at which a hazardous effect is unacceptable. If the economic or social value of the material warrants further consideration, then longer term analyses would be indicated.

\section{REFERENCES}

Cramer, G. M., Ford, R. H. \& Hull, R. L. (1978). Fd Cosmet. Toxicol. 16, 255.

McCann, J., Choi, E., Yamasaki, E. \& Ames, B. N. (1975). Proc. natn. Acad. Sci. U.S.A. 72, 5135 .

Salunkhe, D. K. \& Wu, M. T. (I977). CRC Critical Reviews in Food Science and Nutrition, p. 265.

Waitt, A. W. (1975). Pesticide Science 6, 199. 